
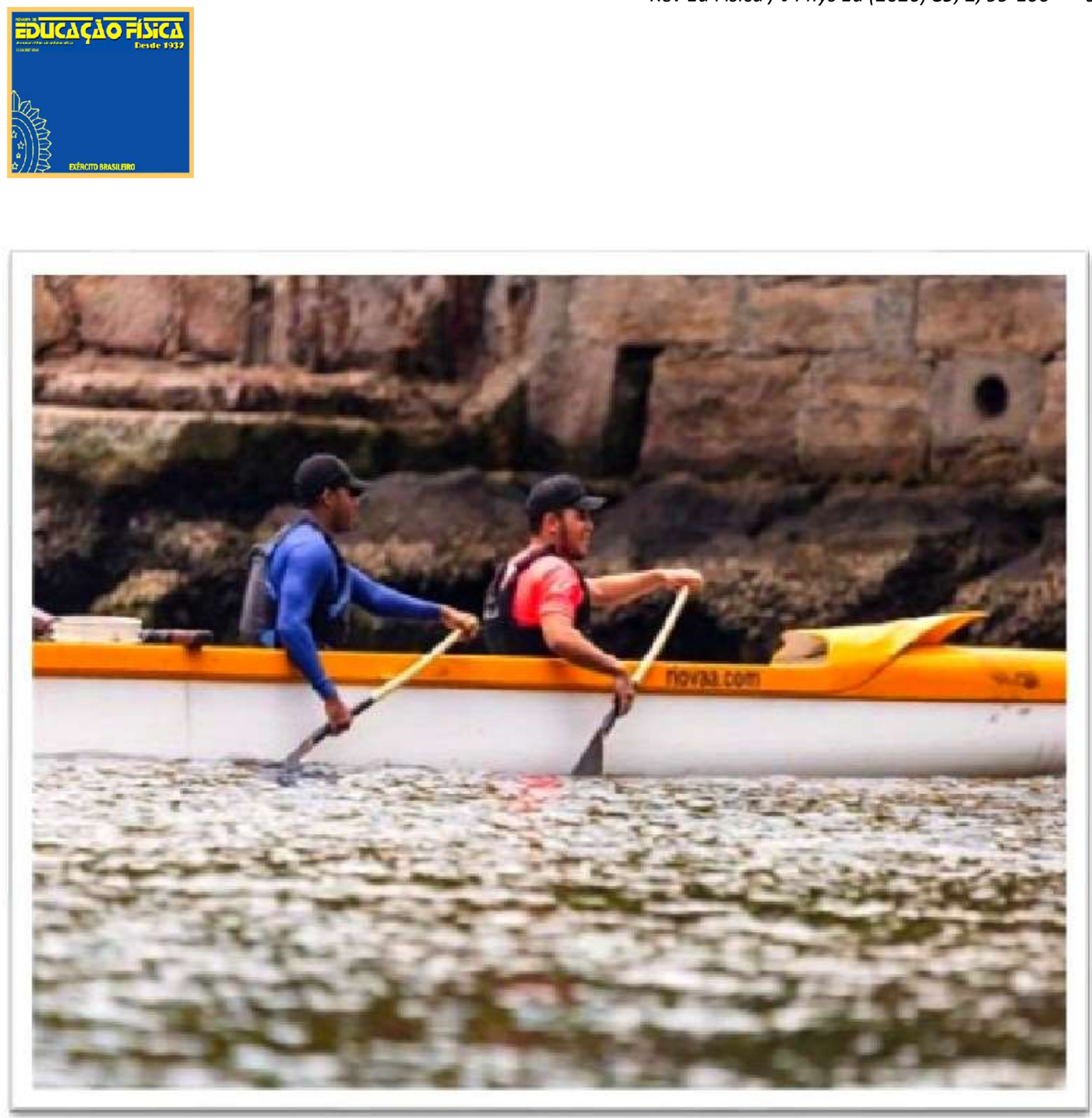

Comentário / Commentary

\title{
O Projeto João do Pulo no Centro de Capacitação Física do Exército (CCFEx) - Experiências iniciais de um núcleo piloto
}

\section{The Brazilian Army Center for Physical Training (CCFEx) “João do Pulo Project" - Initial Experiences at the Pilot Unit}

Mainenti et al. (2020) 
Artigo Original

Original Article

\section{o Projeto João do Pulo no Centro de Capacitação Física do Exército (CCFEx) - Experiências iniciais de um núcleo piloto}

\section{The Brazilian Army Center for Physical Training (CCFEx) "João do Pulo Project" - Initial Experiences at the Pilot Unit}

Míriam Raquel Meira Mainenti ${ }^{\S 1} \mathrm{PhD}$; Angela Nogueira Neves ${ }^{1} \mathrm{PhD}$; Leonardo Stambovsky Guimarães Borges ${ }^{1}$; Luiz Henrique Gomes Fonseca ${ }^{1}$; José Augusto Glycério de Castro ${ }^{2}$

Recebido em: 18 de setembro de 2020. Aceito em: 06 de novembro de 2020.

Publicado online em: 29 de novembro de 2020 .

DOI: $10.37310 /$ ref.v89i2.2685

\section{Resumo}

Introdução: Em 2015, o Ministério da Defesa instituiu um projeto para promover a valorização pessoal e a integração social de militares com deficiência a partir da prática esportiva. Foram estabelecidos quatro núcleos piloto nas três Forças: Marinha, Exército e Aeronáutica, para iniciar o processo e, posteriormente, apoiar novos núcleos.

Objetivo: Apresentar e comentar, em linhas gerais, as propostas do Projeto João do Pulo (PJP) e as experiências iniciais de sua implantação no Centro de Capacitação Física do Exército (CCFEx).

Conclusão: O PJP no CCFEx conta com uma equipe composta por: gerente, coordenador técnico científico, profissionais de saúde (do próprio centro e de instituições parceiras) para monitoramento da saúde, planejamento e execução dos treinamentos físicos e desportivos. A infraestrutura física do CCFEx dispõe de sala de musculação, pista de atletismo, ginásio e laboratórios de pesquisa científica da Escola de Educação Física do Exército (EsEFEx), e do Instituto de Pesquisa da Capacitação Física do Exército (IPCFEx), organizações militares subordinadas ao CCFEx, que complementam as avaliações de saúde dos participantes. As parcerias formadas com instituições que já trabalham com a prática esportiva para pessoas com deficiência têm sido de grande importância para a condução do projeto. Em consonância com os objetivos do Ministério da Defesa para o PJP, espera-se que outras organizações militares do Brasil sejam voluntárias para implementar o PJP, preservando os

Pontos-Chave

- O objetivo do Projeto João do Pulo é promover a valorização pessoal e a integração social a partir da prática esportiva de militares que adquiriram deficiência.

- Apresentam-se, neste, linhas gerais quanto a: recursos humanos, fluxograma de atividades e construção do protocolo de avaliações. - Uma perspectiva futura propõe a inclusão, além de militares, pessoas com deficiência ligadas a instituições parceiras, a fim de promover a integração entre o meio civil e o militar. objetivos primários do projeto e considerando a importância do monitoramento da saúde e do bem-estar dos participantes, bem como, a relevância da realização de parcerias locais, a fim de vencer eventuais obstáculos à sua realização no que se refere à infraestrutura disponível em cada organização militar.

Palavras-chave: reabilitação; pessoas com deficiência; esporte para pessoas com deficiência; pessoal das Forças Armadas.

$\S$ Autor correspondente: Míriam Raquel Meira Mainenti - e-mail: miriam.mainenti@hotmail.com Afiliações: ${ }^{1}$ Escola de Educação Física do Exército (EsEFEx), Rio de Janeiro-RJ, Brasil; ${ }^{2}$ Comissão de Desportos do Exército (CDE), Rio de Janeiro-RJ, Brasil. 


\section{Abstract}

Introduction: In 2015, the Ministry of Defense instituted a program aiming to promote the human valuing and social integration of military personnel with disabilities through sports practice. Four pilot centers were established at the Armed Forces: Navy, Army and Air Force, to start the process and, subsequently, support new centers.

Objective: To present and comment, in general lines, the proposals of the João do Pulo Project (PJP) and the initial experiences of its implementation at the Army Physical Training Center (CCFEx).

Conclusion: The PJP at CCFEx has a team composed of manager, scientific technical coordinator, health professionals (from the center and partner institutions) for health monitoring, planning and execution of physical and sports training. The physical infrastructure of CCFEx has a weight room, athletics track, gymnasium and scientific research laboratories from the Army Physical Education School (EsEFEx), and the Army Physical Training Research Institute (IPCFEx), CCFEx's subordinate military organizations, to complement the participants' health assessments. The partnerships formed with institutions that previously work with sports for people with disabilities are of major importance to the project. In line with the objectives of the Ministry of Defense for the PJP, it is expected that other military organizations in Brazil volunteer to implement the PJP, regarding the primary objectives of the project and consider the importance of monitoring the health and wellbeing of the participants, and the relevance of the realization of local partnerships as well, aiming to overcome eventual obstacles related to the available infrastructure in each military organization.

Keywords: rehabilitation; disabled persons; sport for persons with disability; Armed Forces personnel.

\section{Keypoints}

- The objective of the João do Pulo Project is to promote human valuing and social integration based on the sports practice for military personnel who have acquired disabilities.

- We present here general guidelines regarding human resources, activities flowchart and the structuration of the evaluation protocol.

- Future perspective proposes military personnel, people with disabilities linked to partner institutions, to promote the integration between the civil and the military environment. to include, in addition to the

\section{O Projeto João do Pulo no Centro de Capacitação Física do Exército (CCFEx) - Experiências iniciais de um núcleo piloto}

O Exército Brasileiro (EB) é a força terrestre dentre as Forças Armadas (FFAA) brasileiras, cujas atribuições constitucionais incluem a defesa da Pátria e a garantia dos poderes republicanos, entre outras(1). O risco de vida e a disponibilidade permanente são características da profissão militar, para cujo desempenho se requer o vigor físico(2). Devido a tais características ocupacionais, relacionadas aos equipamentos (armamentos e demais materiais) utilizados, observa-se que há riscos de acidentes, lesões e mortes presentes tanto na preparação quanto no emprego da tropa.

Com vistas a recuperar a saúde física, mental e o bem-estar de militares lesionados em guerras, os Estados Unidos foram o país pioneiro na elaboração de projetos de reabilitação com a criação dos programas Wounded Warriors, em 2003 e Paralympic Military Program, em 2008(3). Canadá, Grã-Bretanha e Austrália seguiram este caminho criando, respectivamente, os programas Soldier on Battle Back e Australian Defense Force Paralympic Sport Program(3).

Felizmente, o Brasil é um país predominantemente pacífico e não tem estado envolvido em conflitos armados internacionais, exceto em missões de paz(4), nas quais destaca-se por sua honra e efetividade(5). Nesse sentido, a prevalência de brasileiros lesionados nessas missões, em relação aos militares de outros países 
que estão em conflitos internacionais, é bastante reduzida. No entanto, o Ministério da Defesa (MD) brasileiro, preocupado com a saúde e o bem estar de seus integrantes que necessitam de reabilitação física e a fim de promover a inclusão social de seu pessoal, desenvolveu o Projeto João de Carlos de Oliveira "João do Pulo"(6,7), conhecido como Projeto João do Pulo (PJP). Instituído pelo MD, foi viabilizado pelas FFAA, cujo objetivo principal estabelecido é o de "promover a valorização pessoal e o fortalecimento da integração social por meio do esporte, no âmbito das FFAA"(6).

O ineditismo da proposta apresentou, aos diversos centros de capacitação física e esportes das FFAA, um desafio para o delineamento quanto a implementação e avaliação de sua efetividade. Na cidade do Rio de Janeiro, há três núcleos do Projeto João do Pulo (PJP): o núcleo Centro de Educação Física Almirante Adalberto Nunes (CEFAN), da Marinha; o núcleo Universidade da Força Aérea (UNIFA), da Aeronáutica; e o núcleo do Centro de Capacitação Física do Exército (CCFEx). O presente comentário focaliza este último núcleo, tendo como objetivo apresentar e comentar, em linhas gerais, as propostas do Projeto João do Pulo (PJP) e as experiências iniciais de sua implantação no núcleo no CCFEx. Cumprir esse objetivo é de suma importância para $\mathrm{O}$ desenvolvimento do PJP. Mesmo com possibilidades de infraestrutura física e de pessoal diversificadas pelo Brasil, os exemplos do núcleo piloto poderão ser um norte para a implantação do projeto nas demais organizações militares em todo Brasil, voluntárias a desenvolver o PJP.

\section{Recursos humanos}

Para a implementação do Projeto João do Pulo, o núcleo CCFEx (PJP-CCFEx) conta com um gerente executivo, militar designado pela Comissão de Desportos do Exército (CDE), responsável pelas questões administrativas, e uma coordenação técnico-científica, designada pela EsEFEx, para planejamento de treinamento físico e esportivo, avaliações periódicas e capacitação de pessoal. A coordenação técnico-científica é responsável pela elaboração do planejamento e supervisão de sua execução. Profissionais de Educação Física tanto do CCFEx quanto de instituições parceiras aplicam o treinamento físico e esportivo. Dentre as instituições parceiras nesses primeiros anos de projeto, destacam-se a Rio Va'a e o Instituto de Educação para Cegos Benjamim Constant.

\section{Fluxo de atividades}

$\mathrm{O}$ gerente do PJP-CCFEx é quem recepciona o militar a tornar-se participante do projeto. Após a verificação das possibilidades de engajamento do militar com deficiência às atividades do PJPCCFEx, agenda-se uma avaliação pré participação com um médico, preferencialmente, da Escola de Educação Física do Exército (EsEFEx), local de realização do treinamento físico rotineiro. Após sua liberação, a coordenação técnico científica, agenda as avaliações funcionais gerais e, adicionalmente, identifica e agenda avaliações específicas às peculiaridades da deficiência apresentada. Em seguida, os treinamentos neuromuscular, cardiopulmonar, funcional e desportivo são planejados e executados. A cada seis meses, realiza-se o protocolo de avaliações para acompanhamento do participante do projeto (Figura 1).

\section{Construção do protocolo de avaliações}

O planejamento elaborado prevê "Avaliações funcionais e de saúde", a cada seis meses. Isto porque há a necessidade de se avaliar se os objetivos propostos em portarias no âmbito Ministério da Defesa (MD) para o PJP têm sido atingidos. Assim, em 2017, a coordenadora técnico-científica do PJP-CCFEx, montou um grupo de pesquisa e conduziu um estudo com o objetivo de construir o protocolo de avaliações para o PJP-CCFEx.

Inicialmente, foram realizadas visitas presenciais e contatos por telefone ou email com instituições e projetos que trabalham com serviços ou com a temática de reabilitação por meio do esporte para pessoas com deficiência física. Após os contatos, as seguintes instituições concordaram em colaborar com a pesquisa: 


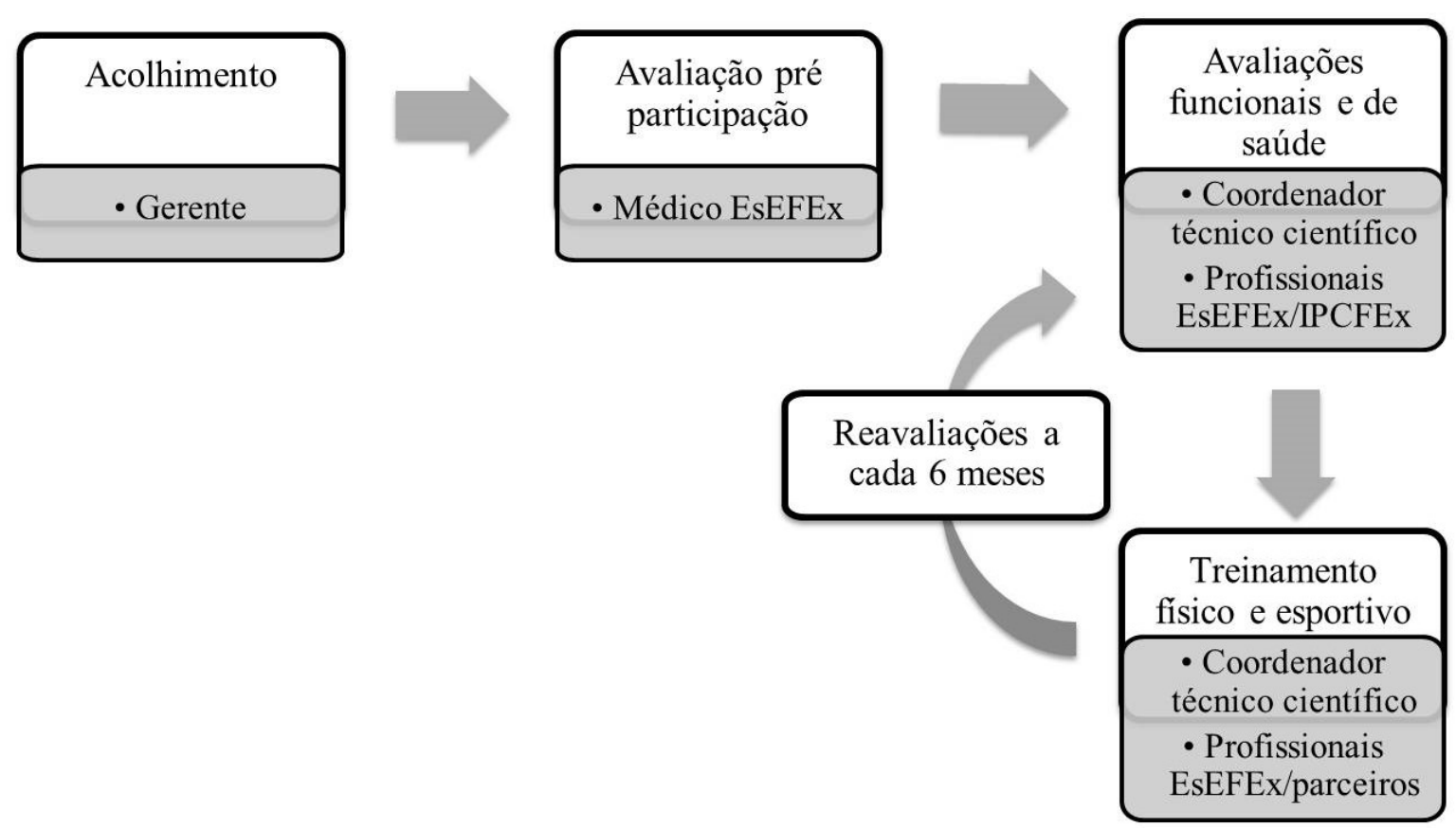

Figura 1 - Fluxograma das atividades desenvolvidas no Projeto João do Pulo, núcleo CCFEX (PJP-CCFEx).

Associação Vencedores Adaptados; Centro Universitário Augusto Motta; Instituto Novo Ser; Associação Niteroiense dos Deficientes Físicos; Projeto Renascer, Servir e Proteger; e Rede Luci Montoro.

O processo de discussão do protocolo com essas instituições seguiu as seguintes etapas: 1) A coordenação técnico-científica propôs uma lista de testes físicos e laboratoriais, a fim de avaliar determinadas capacidades físicas e fisiológicas; 2) Representantes das instituições que concordaram em colaborar responderam dicotomicamente ( $\operatorname{sim} /$ não) se achavam pertinentes os testes sugeridos; 3) Em seguida, eram apresentados os testes para cada capacidade investigada, utilizados nas próprias instituições; 4) Debate com as instituições comparando-se os testes propostos pela coordenação técnico-científica com os utilizados nas instituições: um teste era incluído quando a maioria das instituições identificava como teste pertinente; um teste era modificado quando a maioria das instituições o identificava como não pertinente; outras avaliações foram acrescentadas quando as discussões com os representantes das instituições levaram a sugestões adicionais; e 5) A coordenação técnico-científica fez uma compilação, definindo os testes escolhidos para integrarem as avaliações do PJP, de acordo com o debate com as instituições.

Ao final da pesquisa, o protocolo ficou constituído das seguintes avaliações biopsicossociais: 1) Exames laboratoriais (hemograma, lipidograma, glicose); 2)Composição corporal (somatório de dobras cutâneas, perímetros, e absorciometria por raio-X de dupla energia: DXA); 3) Percepção de qualidade de vida (utilizando o questionário da Organização Mundial de Saúde, específico para pessoas com deficiência física, o WHOQOL-DisPD); 4) Flexibilidade (utilizando o flexiteste); 5) Capacidade cardiorrespiratória (pelo teste de ergoespirometria ou teste de Franklin); 6) Força de membros: superiores (1RM supino e preensão manual) e inferiores (1RM leg press); 7) Potência de membros inferiores (saltos vertical e horizontal); e 8) Potência de membros superiores (arremesso de medicinebol). Para o aprofundamento no estudo 
brevemente apresentado, sugere-se leitura adicional no estudo de Borges(8).

\section{Aplicação do protocolo de avaliações}

Em 2018, o protocolo elaborado foi aplicado pela primeira vez para se avaliar se o projeto estava atingindo os objetivos traçados pelo MD para o projeto(6). Observou-se que o protocolo de avaliações requeria um tempo elevado para sua aplicação. Assim, com o intuito de se evitar que o participante perdesse o interesse no projeto, as avaliações foram reduzidas para as seguintes: perfil bioquímico (hemograma, lipidograma e glicose); composição corporal pelo DXA; e percepção da qualidade de vida relacionada à saúde (WHOQOL-Bref), com o módulo específico para pessoa com deficiência física (WHOQOL-Dis-PD), dos quais alguns itens foram utilizados para construir as avaliações valorização pessoal e integração social, além de uma entrevista.

\section{Valorização pessoal e integração social}

A valorização pessoal é abordada no contexto do autoconceito e do desenvolvimento pessoal. O autoconceito é definido como a percepção pessoal de diversas características que contribuem para a identidade individual; enquanto $\mathrm{o}$ desenvolvimento pessoal é a melhoria de habilidades e qualidades individuais(9). Por sua vez, a integração social é tratada como um processo de assimilação de um indivíduo a um grupo(9). Este constructo é operacionalmente tratado em uma perspectiva mais aproximada ao conceito de inclusão social, apesar de ser conhecida a diferença entre eles(10). Ambos os propósitos do projeto estão englobados no conceito de qualidade de vida(11) e, dessa forma, para a quantificação da valorização pessoal e da integração social, no âmbito do PJP-CCFEx, utilizou-se domínios e facetas dos instrumentos utilizados para avaliar a qualidade de vida (WHOQOL-Bref: e WHOQOL-Dis-PD). Para compor o escore de valorização pessoal, adota-se a média dos domínios físico, psicológico e nível de independência, do WHOQOL-Bref e a faceta autonomia, do WHOQOL-Dis-PD. Para compor o escore de integração social, consideram-se: o domínio relações sociais, do WHOQOL-Bref e as facetas inclusão e discriminação do WHOQOL-Dis-PD.

\section{Considerações sobre o Treinamento Físico e Desportivo}

O coordenador técnico científico, juntamente com os profissionais envolvidos no PJP-CCFEx, planeja e acompanha a execução do treinamento físico $\mathrm{e}$ desportivo. $\mathrm{Na}$ sala de musculação da EsEFEx são realizados os treinos para a preparação física do militar com deficiência, sempre coerente com a modalidade esportiva que o participante deseja fazer ou já realiza. A coerência relatada inclui a especificidade quanto ao gestual esportivo e à velocidade de execução utilizados na modalidade. A disponibilidade atual é de duas vezes por semana, duas horas cada dia, com flexibilidade para aumentar em caso de necessidade.

Em 2018, um militar que foi altamente assíduo em seus treinos, preencheu os critérios de elegibilidade para ser o foco de discussão do Estudo de Caso publicado na presente edição. Seus treinos são realizados na EsEFEx (neuromuscular) e numa praia próxima ao CCFEx, onde o militar pratica Canoagem. Os demais atletas, civis com deficiência da Rio Va'a, devido à parceria formalizada entre CCFEx e o clube, também recebem orientações da equipe do PJP e compartilham o espaço da sala de musculação da EsEFEx no âmbito da preparação física.

Apesar de não ser o principal objetivo do PJP-CCFEx, a CDE tem como uma de suas atribuições, detectar possíveis talentos desportivos que possam ser aproveitados no desporto de alto rendimento paralímpico e orientar $\mathrm{o}$ treinamento dos(as) atletas identificados(as). Nesse sentido, a CDE auxilia o avanço do militar no mundo paralímpico providenciando, por exemplo, alojamento em quartéis próximos aos locais de competições, quando estas são realizadas fora do Rio de Janeiro. 
Perspectivas Futuras do Projeto João do Pulo

Passos importantes foram dados no sentido de a avaliar a eficácia do projeto, a sistematização e a organização documental do PJP-CCFEx. Não obstante, observa-se que o projeto apresenta, ainda, um grande espaço para crescimento, tanto em quantidade de participantes quanto em determinação do público-alvo principal.

Todo o trabalho realizado no núcleo CCFEx, bem como a documentação realizada por meio do presente Comentário e do Estudo de Caso(12), publicado em outro artigo, contribui com o conhecimento fornecendo subsídios a outras organizações militares, que tenham interesse, para que possam oferecer oportunidades de prática esportiva para o militar com deficiência em qualquer ponto do Brasil. Cabe ressaltar que futuras iniciativas devem preservar os objetivos primários do projeto, a importância de monitoramento dos participantes e a relevância da realização de parcerias locais, respeitando as possibilidades de infraestrutura física e de pessoal de cada organização militar.

Uma perspectiva futura recentemente publicado no Decreto $\mathrm{N}^{\mathrm{o}} 10.085$, de 5 de novembro de 2019, que estabelece que o objetivo é incluir, além de militares, pessoas com deficiência ligadas a instituições parceiras para usufruir do PJP e, dessa forma, cada vez mais, promover a integração entre o meio civil e o militar.

Vida longa ao Projeto João do Pulo!

\section{Agradecimentos}

Os autores agradecem às organizações militares e civis envolvidas na avaliação (Escola de Educação Física do Exército EsEFEx e Instituto de Pesquisa da Capacitação Física do Exército - IPCFEx) e treinamento (EsEFEx e Rio Va'a) dos participantes do Projeto João do Pulo (Núcleo CCFEx). Outra importante organização militar nesse processo foi a Comissão de Desportos do Exército (CDE), que, pela gestão do Projeto, buscou a integração de todas as organizações envolvidas. Por fim, os autores também agradecem, ainda, à Academia Paralímpica
Brasileira, do Comitê Paralímpico Brasileiro (APB/CPB), pelo apoio científico.

\section{Declaração de conflito de interesses}

Não há nenhum conflito de interesses em relação ao presente estudo.

\section{Declaração de financiamento}

Estudo realizado sem financiamento.

\section{Referências}

1. Brasil. Presidência da República, Casa Civil, Subchefia para Assuntos jurídicos. Lei Complementar No 97, de 09 de junho 1999. Dispõem sobre as normas gerais para a organização, o preparo e o emprego das Forças Armadas [Internet]. Brasília, DF; 1999. 2019;1-6. [acesso em 05 de junho de 2019]. Disponível em http://www.planalto.gov.br/ccivil_03/lei s/lcp/lcp97.

2. Exército Brasileiro [homepage na internet]. Características da profissão militar [acesso em 14 mai 2019]. Disponível em: http://www.eb.mil.br/caracteristicas-daprofissao-militar.

3. Brittain I, Green S. Disability sport is going back to its roots: Rehabilitation of military personnel receiving sudden traumatic disabilities in the twenty-first century. Qualitative Research in Sport, Exercise and Health. 2012;4(2):244-64. Disponível em doi: 10.1080/2159676X.2012.685100.

4. Celestino S, Neves A N. Missões de Paz: teoria e dimensão humana. Curitiba: Appris, 2020. 239 p.

5. United Nations. Brazil's enduring contribution to UN Peacekeeping. s.d. Disponível em: [acesso em 06 de outubro de 2020]. Disponível em https://news.un.org/en/gallery/537202.

6. Brasil. Ministério da Defesa. Portaria Normativa No 956 - MD, de 23 de abril de 2015. Institui o projeto para valorização pessoal e integração social por meio do esporte, para militares que adquiriram deficiência física [Internet]. 
Diário Oficial da União. 24 de abril de 2015. [acesso em 10 de abril de 2019]. Disponível em http://www.lex.com.br/legis_26712427 PORTARIA_NORMATIVA_N_956 DE_23_DE_ABRIL_DE_2015.aspx.

7. Brasil. Ministério da Defesa. Portaria No 475, de 16 de maio de 2016. Aprova a Diretriz para o desenvolvimento do Projeto "João do Pulo", no âmbito do Exército Brasileiro, que visa à valorização de pessoal e à integração social por meio do esporte, para militares que adquiriram deficiência física (EB10-D-01.034) [Internet]. [acesso em 10 de abril de 2019]. Disponível em http://www.sgex.eb.mil.br/sistemas/be/ copiar.php?codarquivo $=1442 \&$ act $=$ bre .

8. Borges LSG. Modelo para avaliação dos participantes do Projeto João do Pulo no Exército Brasileiro. [Trabalho de Conclusão de Curso de Bacharelado em Educação Física]. Rio de Janeiro: Escola de Educação Física do Exército (EsEFEx); 2017.

9. VandenBos GR. APA Dictionary of Psychology. 2a Edition. WashingtonDC: APA Books, 2011.

10. Nunes SS, Saia AL, Tavares RE. Educação Inclusiva: Entre a História, os Preconceitos, a Escola e a Família. Psicologia: Ciência e Profissão. 2015;35(4):1106-19. Disponível em doi: 10.1590/1982-3703001312014.

11. The WHOQOL Group. The World Health Organization Quality of Life assessment (WHOQOL): Position paper from the World Health Organization. Social Science \& Medicine. 1995;41(10):1403-9. Disponível em doi: 10.1016/0277-9536(95)00112-K

12. Fonseca, LHG et al. Modificações biopsicossociais de um militar com deficiência aderente ao Projeto João do Pulo (Núcleo CCFEx) - Estudo de caso longitudinal. Revista de Educação Física / Journal of Physical Education. [Online] 2020;89(2):88-98. Available from: doi: 10.37310/ref.v89i2.1525 